\title{
S. J. P. AND VARIOUS DRUGS
}

\author{
KEN NODA \\ Department of Physiology, Kurume University School of Medicine, \\ Kurume-shi, Japan
}

In the investigations of skeletal muscle of frogs the microelectrode study makes it possible to distinguish the tonic fiber from the kinetic or sensory fiber. To the potential obtainable from tonic fibers Kuffler et al. (1) named a small-nerve junctional potential (S. J. P.). In detail, S. J. P., probably originating at a junction from a small motor axon to the tonic fiber, resembles the end-plate potential of twitch fiber because S. J. P. shows no overshoot at action and does not generate the propagatable impulses.

Though there is an opinion that it is naturally to think that S. J. P. and twitch fiber spike do not indicate the presence of two different muscle fibers but express the different styles of nervous innervation on the same muscle fiber, the author has carried out the present experiments, assuming the existence of a slow fiber in skeletal muscle of frogs. A duality of response of a muscle fiber is not considered in the course of these experiments of frog muscles.

The characteristics of the small-nerve junctional potentials were precisely investigated by Kuffler and Vaughan Williams (2) and by Burke and Ginsborg (3). But there is no report on the effect of various drugs on these S. J. P.'s, so the author has demonstrated the effect of externally applied acetylcholine, vagostigmine, sodium fluoride; monoiodoacetic acid, tetraethylammonium chloride, etc. on S. J. P. in order to know whether the transmission of the small-nerve junction is cholinergic or not and to know whether the S.J.P. and its following after potential are caused by the same process or not.

\section{METHODS}

Sciatic-iliofibularis preparation was obtained from frogs through this experiment carried out in all seasons. Only one small branch of sciatic nerve entering into iliofibularis muscle was remained, and the surrounding connective tissue was carefully removed. After immersing these preparations into the Ringer bath, a microelectrode inserted into a muscle fiber records S. J.P. caused by the electrical stimulation to sciatic nerve. Occasionally high frequency stimulation was given to sciatic nerve in order to block the large motor axon.

As shown in later, when one or two $\mathrm{ml}$ of various drugs in respective concentration shows their specific effect, S. J. P. obtained changes in shape of depolarization or in after potential. 


\section{RESULTS AND DISCUSSION}

Small-nerve junctional potentials obtained are shown in Figure 1 in which no overshoot and the presence of positive (downwards) afier potential can be observed. S. J. P. has the depolarization, the repolarization, and the hyperpolarization as its elementary components.
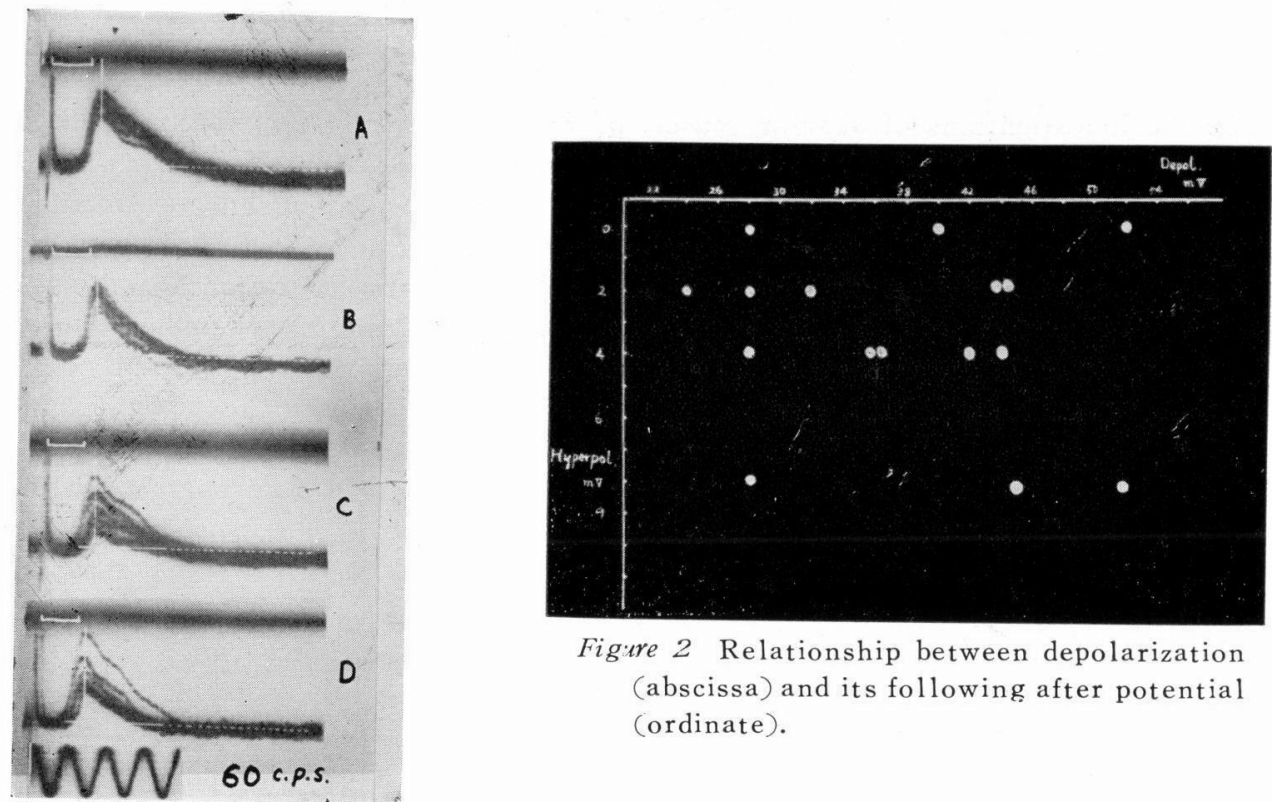

Figure 2 Relationship between depolarization (abscissa) and its following after potential (ordinate).

Figure 1 Four S. J. P.'s. Horizontal bar signifies the zero level of resting potential.

The resting membrane potential of a slow fiber is about $60 \mathrm{mV}$ on the average, and depolarizes in the average value of $37 \mathrm{mV}$, the maximum value of a following hyperpolarization being about $8 \mathrm{mV}$. The relationship between depolarization and hyperpolarization is demonstrated in Figure 2 and indicates that the ratio of hyperpolarization to depolarization is not constant, suggesting the presence of no correlation. Even if the value of depolarization is larger, its following hyperpolarization is not always deeper. No parallelism in hyperpolarization and depolarization presumably indicates no interdependency of both processes. This ratio in the present experiments $(1 / 15$ to $1 / 30)$ is less than that reported by Kuffler et al. (2). The rise and the decay require, on the average, 8 and $36 \mathrm{msec}$. respectively.

Next, the following various drugs cause the change of S. J.P.

(1) Curare $\left(6 \times 10^{-4--6} \mathrm{mg}\right)$

It has been thought that curare does not act upon the electrical properties of postsynaptic membrane. In the author's experiments S. J. P.'s were promptly disappeared 
by applying the curare, though Tasaki (4) reported that S. J. P. was, at last, vanished after the gradual decreasing of the potential. Curare is, also, effective to block S. J. P. (Figure 3).
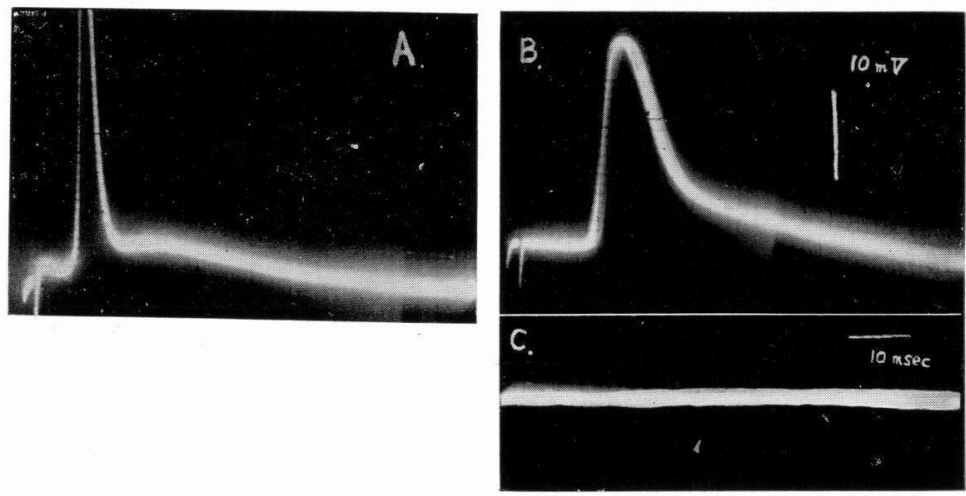

Figure 3 Spike potential of twitch fiber (A) and S. J.P. (E) obtained from the same muscle, and the disappearance of S. J.P. (C) by curare.

a

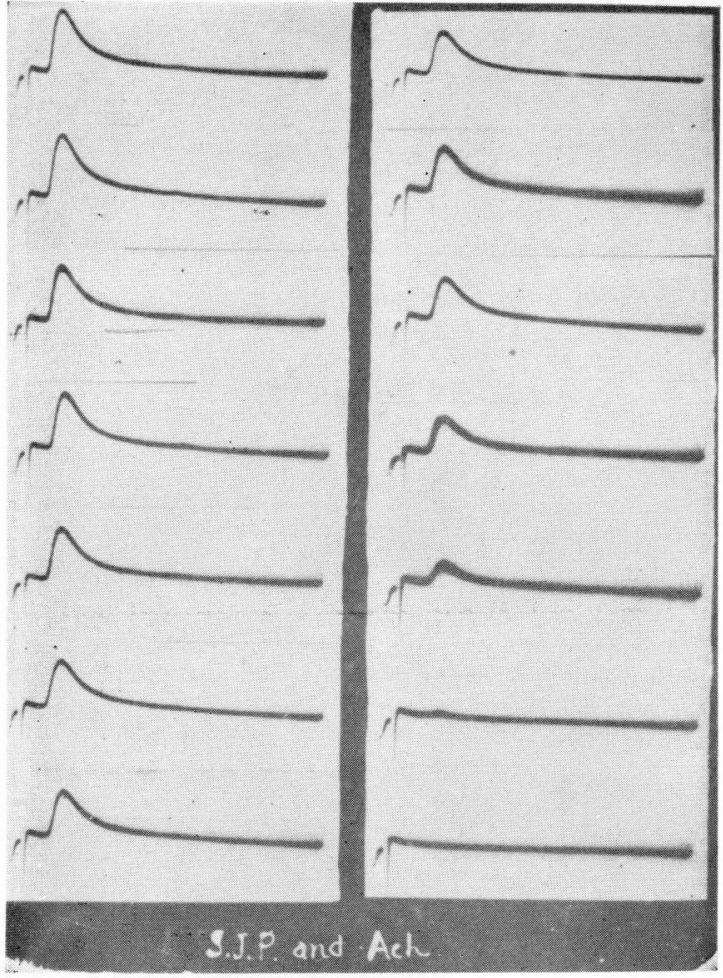

Figure 4 a: S.J.P. without any treatment. S.J.P.'s below b are recorded for about ten minutes after treating with asetylcholine.
(2) Acet ylcholine $\left(10^{-5}\right)$

Grundfest (5) described that the potential of slow muscle fiber is a postsynaptic potential which is only chemically excitable, and that the electrogenesis is not determined by the excitant but by the property of synaptic membrane. On the other hand, it is well known that the transmission of twitch fiber junction is performed by an acetylcholine. Therefore, it is a problem to determine whether the transmission of junction of slow fiber is cholinergic or not. For this purpose, this procedure has been carried out.

When the iliofibularis muscle showing S. J. P. is treated by $10^{-5}$ acetylcholine solution, the latency, the depolarization height and even the hyperpolarization remain unchanged for about ten minutes. Then S.J.P. begins to diminish with losing the hyperpolarization (Figure 4). 
The duration of about ten minutes described above is extremely longer than that requiring for showing the effect of any other drugs upon S. J.P. So, it may be thought acetylcholine does not primarily act upon the cellular element generating S. J. P.'s. Moreover, the size of after potential remains unchanged longer than the decrease of depolarization; namely, the after potential resists to an action of acetylcholine more than the depolarization, this tendency being also observable in the experiments of vagostigmine.

\section{(3) Vagostigmine $\left(10^{-5}\right)$}

As shown in Figure 5, the effect of vagostigmine is analogous to that of acetylcholine upon S. J.P. No influence upon both latency and depolarization and the presence of after potential resistant more than depolarization are similarly observed in the experiments of vagostigmine as well as in those of acetylcholine. The very slight difference present between the effect of vagostigmine and that of acetylcholine is that S. J. P. treated by vagostigmine begins to decrease earlier than that by acetylcholine.

These facts presumably indicate that S. J. P. is uncholinergically excitable when it is generated by the stimulus via nerve. If the transmission by acetylcholine

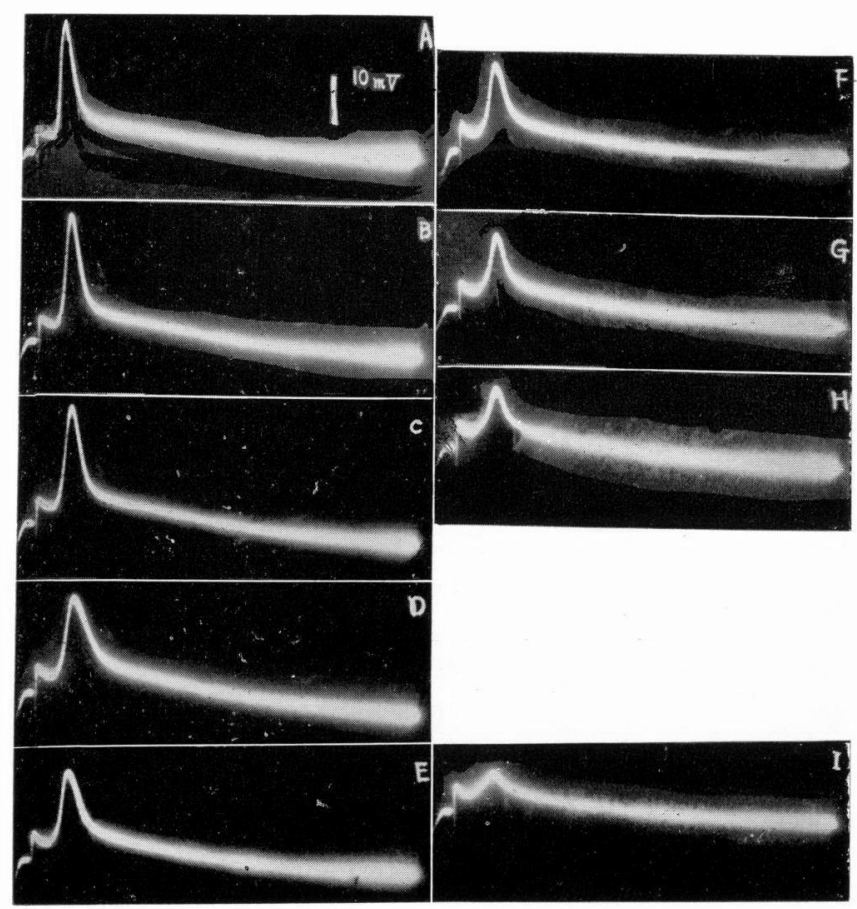

Figure $5 \mathrm{~A}$ is a control S.J.P. S. J.P. below B is obtained after treatment by vagostigmine, for about five minutes.

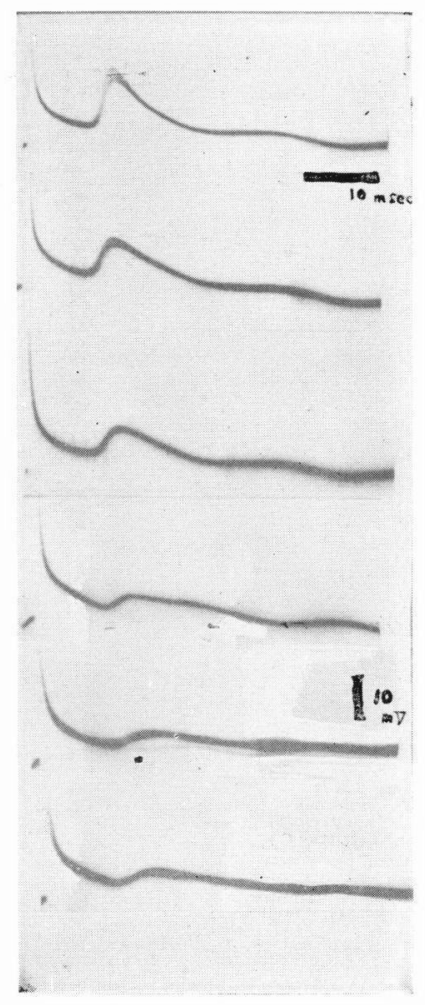

Figure 6 S.J.P. and TEA-Cl. All traces were recorded during two minutes. 
has been consistently supported by the thesis that the slow fiber responds remarkably to this substance, above consideration may be accurate. Eccles, J. C. (6) states that so far as it has been investigated the pharmacology of small-nerve junctional potential is similar to that of spike potential of twitch fiber. The synapse is not electrically excitable (Grundfest) and it must, therefore, be evoked by a chemical effect of presynaptic activity. The author's experiments, however, demonstrate that acetylcholine has no so direct relation to the transmission of small-nerve junction.

(4) Tetraethylammonium chloride $\left(10^{-4}\right)$

The effect of TEA-Cl upon S. J. P. is shown in Figure 6 in which no change of latency and the gradual early decrease of depolarization can be observed. This may be caused by the blocking action of TEA ion rather than by the evoking action of that ion to spontaneous discharge of endplate.

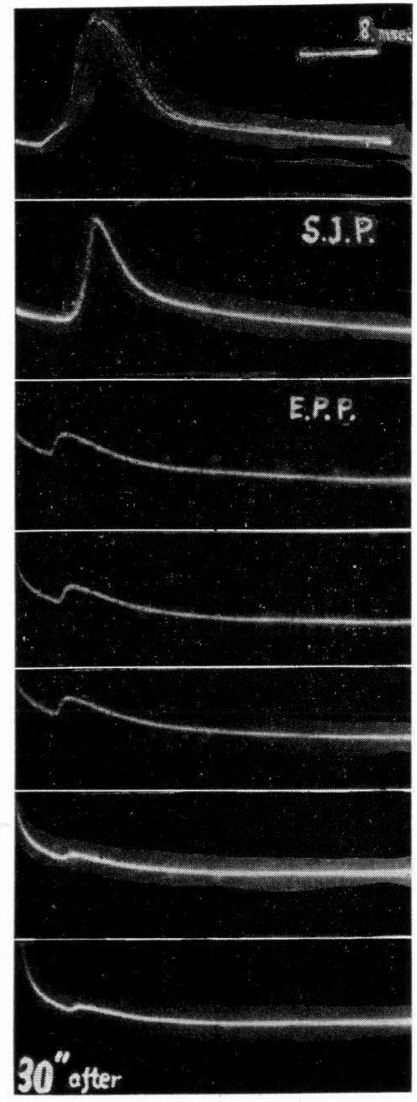

Figure 7 E. P. P. and DNP. Lower five traces show the change of E.P.P. to DNP (down wards). The uppermost and the second trace are a spike of twitch fiber and S. J. P. obtained from the same muscle.

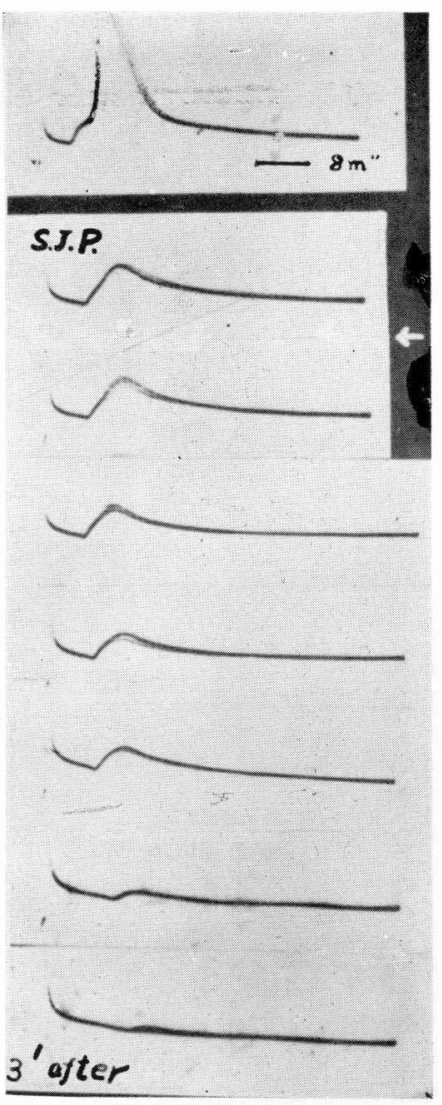

Figure 8 S. J.P. and DNP. Arrow signal indicates the moment of DNP treatment. 


\section{(5) 2.4-dinitrophenol (3 $\mathrm{mM})$}

Dinitrophenol is a metabolic oxidative inhibitor. When the preparation is treated by DNP, the change of E.P. P. and S. J. P. of the same muscle has been observed in order to know the property of postsynaptic membrane of twitch fiber and of slow fiber because both potentials are similarly a postsynaptic potential. DNP depresses the electrical activity of these two potentials until they ultimately cease (Figures 7 and 8 ). Though the fact that the duration till cessation of E.P.P. (about $30 \mathrm{sec}$ ) is shorter than that of S. J. P. (about $5 \mathrm{~min}$ ) may express only the stronger action of DNP upon the postsynaptic membrane of twitch fiber junction, both mechanisms may be different. Namely, the latency of S. J. P. immediately before cessation has prolonged to twice of original duration of latency while that of E.P.P. is constantly till cessation. These facts may suggest the difference in characteristics of postsynaptic membrane of both fibers.

(6) Sodium fluoride $(5 \mathrm{mM})$ and Monoiodoacetic acid (1 $\mathrm{mM})$

By administering NaF and MIAA to S. J. P., an interesting fact in association with the correlation between S. J. P. depolarization and hyperpolarization has been found out.

In this part of the report, the problem of positive after potential (hyperpolarization) is discussed. S. J. P. is followed by a positive (always positive) after potential, but it has sometimes no after potential when the secondary changes in

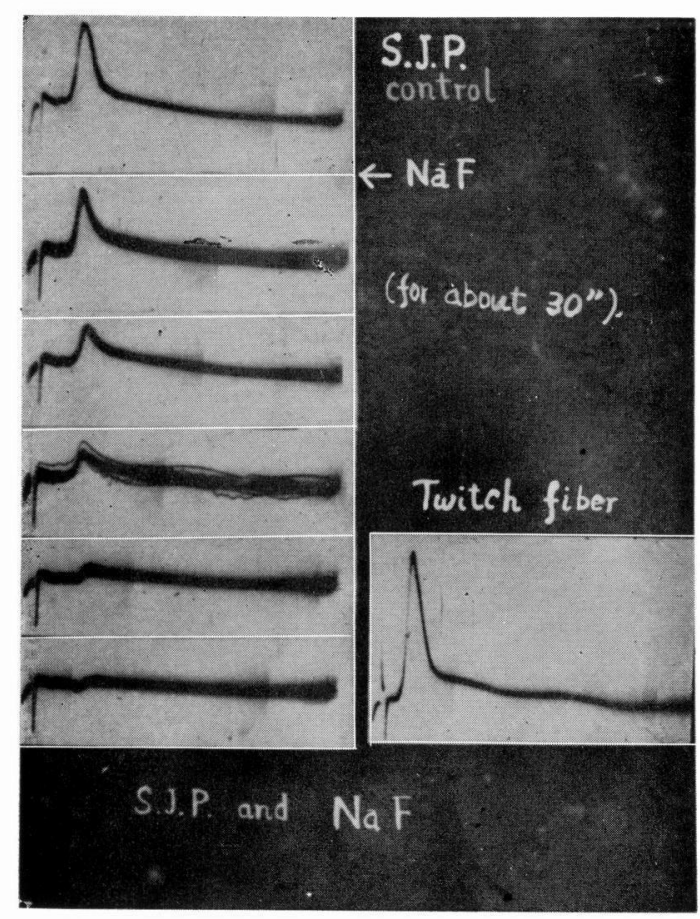

Figure 9 muscle fiber can not be considered. In the twitch fiber, Frank (7) states. both negative after potential which shows various types and resting potential are determined by the same factor. In this paper it has been attempted to distinguish the depolarization of S. J. P. from the after potential, from the metabolic point of view. Probably these experiments make it possible to clarify the fact that S. J. P. which is a synaptic potential is always (or irequently) followed by an after potential.

The external application of $\mathrm{NaF}$ which has the anticholinesterase action and the metabolic inhibiting action resulted in the rapid disappearance of positive after potential when very slight decrease of height of S. J. P. can be seen (Figure 9). Among many biological actions of $\mathrm{NaF}$, the anticholinesterase action is negligible 


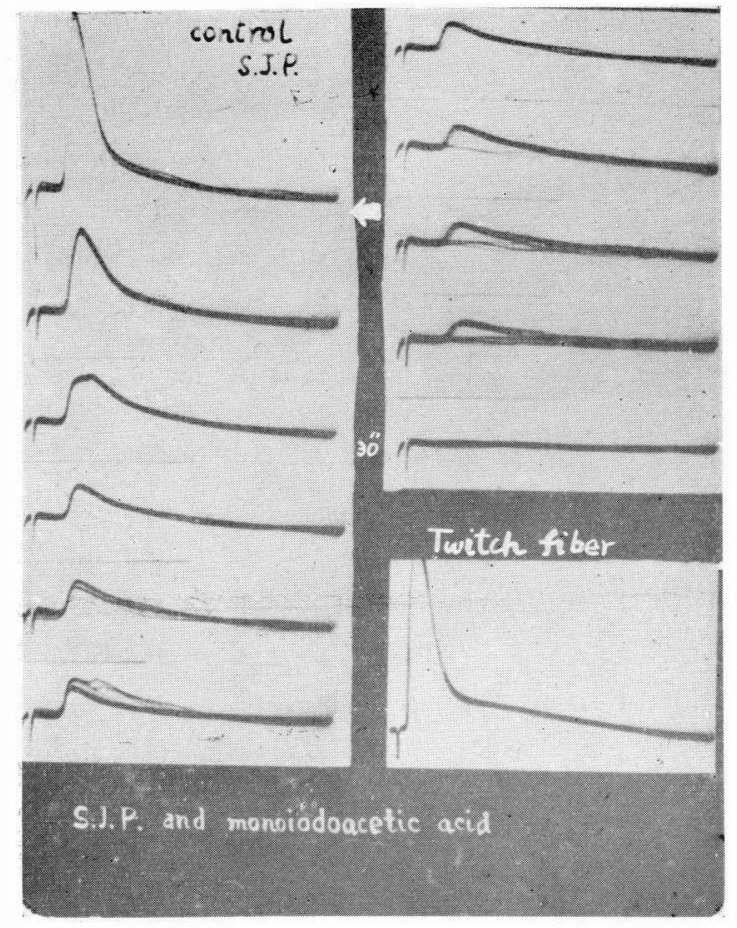

Figure 10 from above experiments by use of acetylcholine or vagostigmine, therefore, this may be due to the metabolic inhibition. Accordingly, it is supposed the positive after potential is more metabolically built up than the S. J. P. depolarization.

On the contrary, even when the height of depolarization has been decreased to very small size (till $1 / 7$ of original size) by MIAA the hyperpolarization remains unchanged. In other words, MIAA affects on depolarization more intensively than on after potential. MIAA causes, also, the slight prolongation of latency. By many experiments with use of MIAA or $\mathrm{NaF}$ it is clarified that the process for depolarization is probably different from that for after potential (Figure 10).

Burke and Ginsborg (3) describe that the diphasic decay of S. J. P. is not due to the specific activity of neuromuscular transmitter, but is probably dependent upon the delayed rectification due to delayed rise in potassium conductance. The difference in attitude of depolarization and after potential to these metabolic inhibitors may be resulted from the difference in electrogenic metabolism of these two processes, the further investigation on this thesis being required.

\section{SUMMARY}

S. J. P., obtained from iliofibularis of frogs and recorded by a microelectrode, changes when various drugs are externally administered to tonic fibers.

(1) Endplate potential which is a synaptic potential and S. J. P. which is probably a synaptic potential show the difference in the duration till disappearance and in the latency (prolongation in S. J. P.), when DNP is applied.

(2) The experiments by use of acetylcholine, vagostigmine or TEA-Cl do not give an active support for the cholinergic transmission of small-nerve junctions, and show that the after potential resists more than the depolarization when S. J. P. becomes smaller.

(3) NaF affects upon the after potential more intensively than upon the depolarization while MIAA is more effective in the disappearance of depolarization than in that of after potential. Accordingly, the depolarization of S. J. P. and the after 
potential of it may have a separate or individual basis of cellular metabolism.

I wish to express my gratitude to Prof. K. Suenaga and Prof. K. Koketsu for their guidance and interest in this study.

\section{REFERENCES}

1. Kuffler, S. W. and Gerard, R. W.; The small-nerve motor system to skeletal muscle. J. Neurophysiol.. 10, 383-394, 1947.

2. Kuffler, S. W. and Vaughan Williams, E. M.: Small-nerve junctional potential. The distribution of small motor nerves to frog skeletal muscle, and the membrane characteristics of the fibers they innervate. J. Physiol. 121. 289-317, 1953.

3. Burke. W. and Ginsborg, B. L.: The electrical properties of the slow muscle fiber membrane. J. Physiol. 132, 586-598, 1956.

4. TASAKI, I.: The action potential at motor end plate. The advance in physiology. (in Japanese) 319-346. 1949.

5. Grundfest, H. : Electrical inexcitability of synapses and some consequences in the central nervous system. Physiol. R. Vol. 37, No. 3, 337-361, 1957,

6. EcCles, J. C. : The neurophysiological basis of mind. The Clarendon Press. 1953.

7. Frank, G. B. : Negative after-potential of frog's skeletal muscle. J. Neurophysiol. Vol. 12, 602-614, 1957. 